\title{
MS23-03 | Multidimensional Aperiodic Structures from BGU
}

Ben-Abraham, Shelomo (Ben-Gurion University of the Negev, Beer Sheba, ISR)

I present an overview of multidimensional deterministic aperiodic structures which I constructed together with my collaborators, mainly my own students. First I present the octagonal two-color Ben-Abraham-Gähler structure constructed by maximal covering of the plane by two octagonal patches. It serves to interpret the structure of the octagonal MnSiAl quasicrystal found by Kuo et al. and investigated by electron crystallography by Hovmöller et al. It is an infinite layer structure with layers alternating in color reflecting the physical structure where alternating layers are antiphases of each other. Next I show our multidimensional generalizations of the Paperfolding and Period doubling sequences. In the sequel I present the Color coding of the chair tiling in arbitrary dimension and the multidimensional Brick tiling (my generalization of the table tiling) as well as its color coding. The latter has a singular continuous part in its Fourier spectrum.Finally I show my two newest structures: The Crossed cube structures - generalizations of the Crossed square tiling whose singular continuous Fourier spectrum is equivalent to that of the non-Pisot squiral tiling.At last I present the multidimensional generalization of the Golay-RudinShapiro sequence whose Fourier spectrum is essentially absolutely continuous. 